\title{
The Fault Location Method of Distribution Network with Fault Indicators Based on Impedance
}

\author{
Xinpei $\mathrm{Hao}^{1}$, Zhaoyong Meng ${ }^{1}$, Wuhao Huang ${ }^{2}, \mathrm{Tao} \mathrm{Tao}^{2}$, Yida Wang ${ }^{2}$ \\ 1.School of Electrical Engineering of ShanDong University, ShanDong, ,250061, China \\ 2.Hangzhou Power Supply Company, ZheJiang, 310000,China
}

Keywords: interpolation. impedance method; fault indicator; distribution network; fault location

\begin{abstract}
The structure of distribution network is complex, and multi-hybrid power lines such as wires and cables are used, which cause difficulties in accurate determination of fault distance. A new method to pinpoint faults was provided by this paper. The fault branch was located with the logic operation of the status information provided by the disconnect switches and wiring fault indicators, and the fault distance was accurately calculated by the impedance method. In this method, the equations were written, in which the fault point and fault positive sequence voltage were treated as the unknown quantity, and the fault distance was calculated by solving the equations. Simulate with ATP, and the data was processed with MATLAB to verify the feasibility of the algorithms in complex distribution network fault location. The results show that the location method has a good localization accuracy.
\end{abstract}

\section{Introduction}

Distribution network fault location methods are mainly impedance method[1,2], traveling wave method[3-5], signal injection method[6], etc. The traveling wave method has the dead zone in the presence of positioning[7], as the multi-branch distribution network is greatly influenced by the reflected wave, and it is susceptible to line type, wiring, etc. Signal injection method requires to inject a specific type of signal into the fault system. The signal is prone to distortion which will cause measurement errors[8], and additional device is needed, which will meet large investment costs.

The impedance method is nothing to do with fault time and system impedance, and there is no dead zone or other issues. Compared with other methods, the technology is relatively mature. The fault indicator with a communication function application make the impedance method have a large advantage, which is able to take full advantage of the disconnect switches and fault indicators installed in distribution lines. According to the feeder terminal status information, fault indicators and the two-phase current and voltage information of trunk lines, fault distance is calculated, which greatly reduces the investment.

When a short circuit fault, from the power line to the fault point, there will be a fault current flow through the Feeder Terminal Unit (FTU) on trunk lines and fault indicators on branch lines. The over-current information and fault information is uploaded to the main station through the communication network. The main station analysis software determines the fault loop and locates the fault zone based on the distribution network topology. With the fault voltage and current information provided by FTU, the impedance between the measurement point and the fault point is accurately calculated.

\section{Fault zone positioning principle}

The FTU have the measuring circuit voltage and current capability to capture voltage and current information on the fault line of the distribution network. Fault indicators only judged whether the line is over-current, and sent the over-current information to the dispatch center. 


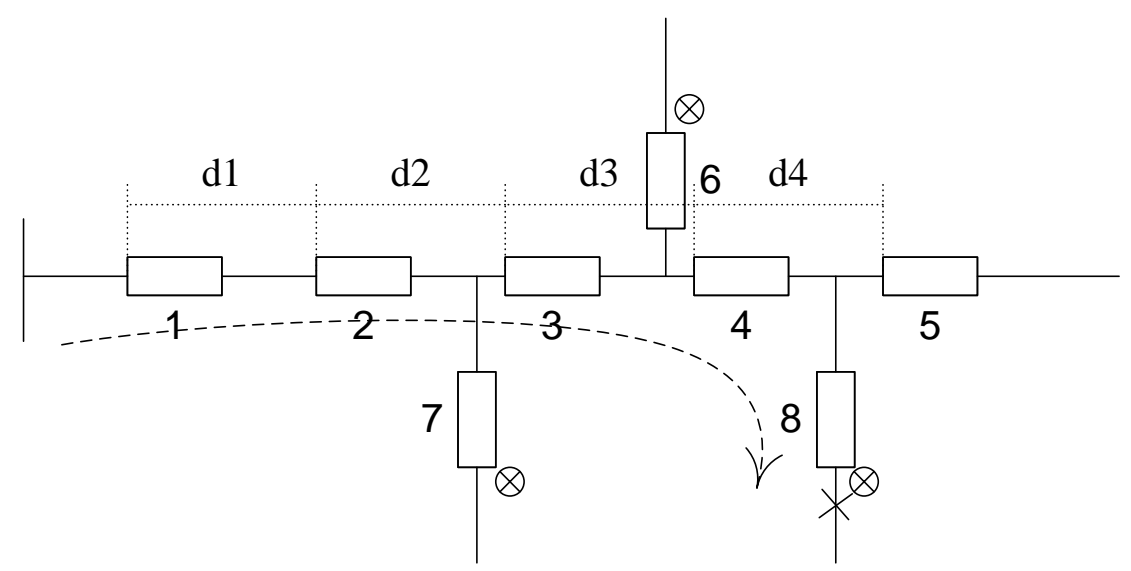

Fig.1 FTU and fault indicators distribution in Distribution network

FTU and fault indicators' distribution in Distribution network is shown in Fig.1. FTU are installed at the trunk line, and the distance between adjacent FTU is d1, d2, d3, d4. Fault indicators are installed at the branch. For example, when phase short circuit fault occurs at branch 8 in Fig.1, there will be fault current flows from the source to the point of fault. That is: Disconnect switches 1-4 and fault indicator 8 will have fault current flows, while others FTU and fault indicators don't have over-current information. The fault intervals can be located with logical judgment.

\section{Phase short circuit ranging principle}

\section{Ranging foundation}

FTU record the information of two-phase AC's voltage and current. When two phase short circuit fault occurs there is no zero sequence component, and the two phase short circuit current value equal but opposite in directions. It can be launched:

$$
\begin{aligned}
& \dot{U}_{b}=-\left(\dot{U}_{a}+\dot{U}_{c}\right) \\
& \dot{I}_{b}=-\dot{I}_{a} \text { or }-\dot{I}_{c}
\end{aligned}
$$

$U_{a}, U_{b}, U_{c}$ is the volatge of A, B,C phase. $I_{a}, I_{b}, I_{c}$ is the current of A, B, C phase. According to the boundary conditions, when metallic short circuit occurs in the feeder, the positive sequence voltage is 0.5 times of the non-fault phase voltage at the fault point[9]. Positive sequence voltage can be represented by non-fault phase:

$$
\dot{U}_{k a 1}=0.5 \dot{U}_{k a}
$$

$U_{k a 1}$ is the positive sequence voltage at the fault point; $U_{k a}$ is the non-fault phase voltage at the fault point. The positive sequence voltage can be represented by the voltage and current at the measuring point:

$$
\dot{U}_{k a 1}=0.5\left(\dot{U}_{i a}-\dot{I}_{i a} \sum_{i=1}^{n} Z_{i} d_{i}\right)(i=1,2 \cdots n)
$$

$U_{i a}$ and $I_{i a}$ is the positive sequence voltage and current at the $i-t h$ measuring point and $d_{i}$ is the distance between adjacent measurement points.

\section{DFT algorithm}

Discrete Fourier Transform (DFT) has a complete digital signal analysis and processing method, which simplifies the process of discrete data. It is applicable to discrete data processing in distribution network fault. 


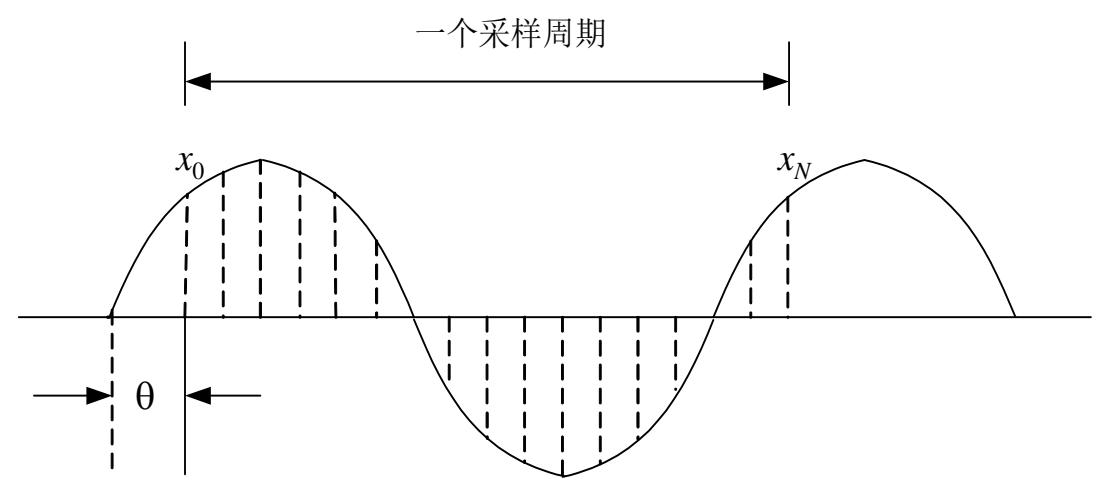

Fig.2 Schematic of Sampling

The schematic of sampling is shown in Fig.2. When a fault occurs, FTU sample two-phase voltage and current, which will produce a large number of discrete sampling data. Discrete sampling data is converted to voltage and current vector by DFT. The fault distance can be accurately calculated by using the voltage and current vector.

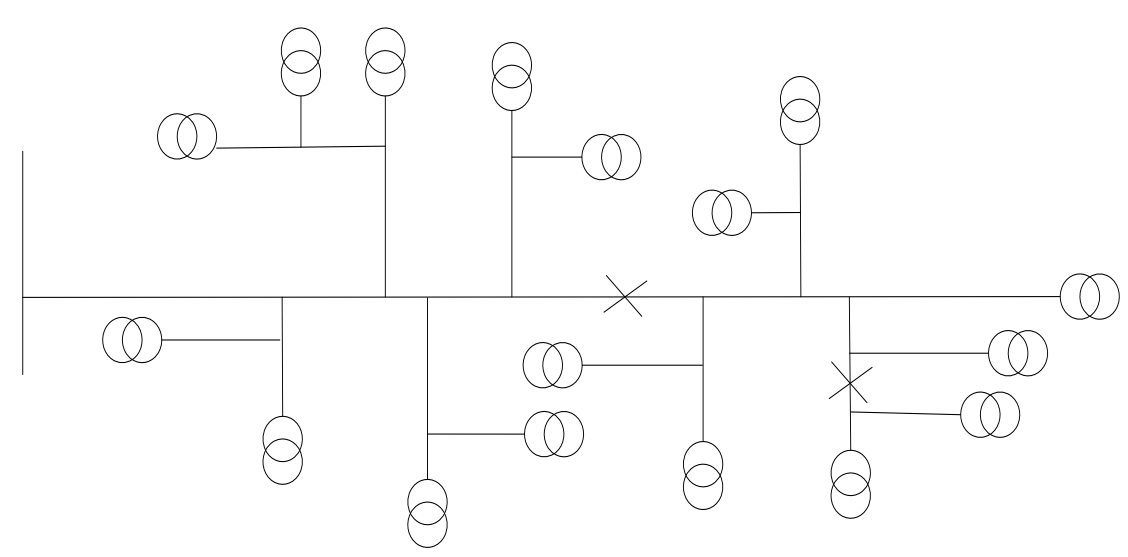

Fig.3 Structure of Simulation Model

According to the DFT method, the sampling values are substituted into the DFT formula. The voltage and current waveform function can be available:

$$
\dot{X}=X_{S}+j X_{C}=\frac{\sqrt{2}}{N}\left[\sum_{k=0}^{N-1} x_{k} \sin \left(\frac{2 \pi}{N} k\right)+j \sum_{k=0}^{N-1} x_{k} \cos \left(\frac{2 \pi}{N} k\right)\right]
$$

$N$ is the number of samples in one cycle. $k$ is The $k-t h$ sample value.

\section{Establish ranging expression}

When the phase fault occurs, in which the position showed in Fig.1. Disconnect switches 1-4 have fault current flows. FTU offer two-phase voltage and current information. Write the fault equation with the positive sequence component:

$$
\dot{U}_{i a 1}-\dot{U}_{k a 1}=\dot{I}_{i a 1} \sum_{i=1}^{n} Z_{i} d_{i}(i=1,2,3 \cdots n)
$$

$\dot{U}_{i a 1}$ and $\dot{I}_{i a 1}$ is positive sequence voltage and positive sequence current at the measurement point.

When non-metallic phase short circuit fault occurs, the short circuit resistance is $r$. Write the composite sequence network fault equation:

$$
\dot{U}_{i a 1}-\dot{U}_{k a 1}=\frac{1}{2} \dot{I}_{i a 1} r+\dot{I}_{i a 1} \sum_{i=1}^{n} Z_{i} d_{i}(i=1,2,3 \cdots n)
$$


Combined formula (6) with (7), and independently represents the last section near the end of the fault line:

$$
\begin{aligned}
\dot{U}_{i a 1}-0.5 \dot{U}_{i a} & +0.5 \dot{I}_{i a} \sum_{i=1}^{n-1} Z_{i} d_{i}-\dot{I}_{i a 1} \sum_{i=1}^{n-1} Z_{i} d_{i}=\frac{1}{2} \dot{I}_{i a 1} r \\
& +\left(\dot{I}_{i a 1} Z_{n}-0.5 \dot{I}_{i a} Z_{n}\right) d_{n}(i=1,2,3 \cdots n)
\end{aligned}
$$

Order:

$$
\left\{\begin{array}{l}
T=\frac{1}{2} \dot{I}_{i a 1} \\
Q=\dot{I}_{i a 1} Z_{n}-0.5 \dot{I}_{i a} Z_{n} \\
H=\dot{U}_{i a 1}-0.5 \dot{U}_{i a}+0.5 \dot{I}_{i a} \sum_{i=1}^{n-1} Z_{i} d_{i}-\dot{I}_{i a 1} \sum_{i=1}^{n-1} Z_{i} d_{i}
\end{array}\right.
$$

Then: Formula (10) can be rewritten as:

$$
H=Q d_{n}+\operatorname{Tr}
$$

To eliminate the fault resistance effects of non-metallic fault, multiplied the conjugate ${ }^{*}$ of $T$ on both sides of formula (14) at the same time:

$$
\stackrel{*}{T} H=\stackrel{*}{T} Q d_{n}+\stackrel{*}{T} T r
$$

Both sides take the imaginary part at the same time, the fault distance can be accurately calculated:

$$
d_{n}=\frac{\operatorname{Im}\left(H T^{*}\right)}{\operatorname{Im}\left(Q T^{*}\right)}
$$

\section{Verify ranging algorithm}

\section{ATP simulation}

A distribution line model in $10 \mathrm{kv}$ voltage level is build with ATP. The simulation uses hybrid line. The total length of trunk line is $5 \mathrm{~km}$, and positive sequence impedance is $0.17+\mathrm{j} 0.402 \mathrm{ohms}$ per km; The length of the branch line from left to right is $1 \mathrm{~km}, 0.5 \mathrm{~km}, 1.2 \mathrm{~km}, 0.8 \mathrm{~km}, 1 \mathrm{~km}, 1.5 \mathrm{~km}$, and positive sequence impedance is $0.65+\mathrm{j} 0.412 \mathrm{ohms}$ per $\mathrm{km}$. Phase faults are set on the trunk lines and branch lines. Fault information collected is the line over-current information and two-phase line voltage and current information. The specific simulation model is shown in Fig.3.

\section{Results verify}

The two-phase voltage and current information generated by ATP simulation is imported into MATLAB to process the fault data. The discrete voltage and current sampling information is converted into voltage and current vectors. The fault distance is calculated by using the data located in different positions of the distribution lines to reduce accidental errors caused by data acquisition.

When the trunk lines occurs two-phase metal short circuit, the results of distance measurement are shown in Table 1. It shows that with the distance between FTU and fault decrease the fault range accuracy gradually increased. This is due to the fault current of non-fault branch is ignored in the calculation. It belongs to the theoretical error and cannot be eliminated. 
Tab.1 Fault location results of trunk line with metal short circuit

\begin{tabular}{|l|l|l|l|l|l|l|}
\hline $\begin{array}{l}\text { fault } \\
\text { distance(km) }\end{array}$ & FTU1 & FTU2 & FTU3 & FTU4 & $\begin{array}{l}\text { absolute } \\
\text { error(km) }\end{array}$ & $\begin{array}{l}\text { relative } \\
\text { error (\%) }\end{array}$ \\
\hline 1.0 & 1.0564 & 1.0089 & & & 0.0089 & 0.890 \\
\hline 1.5 & 1.5974 & 1.5119 & & & 0.0119 & 0.793 \\
\hline 2.0 & 2.1588 & 2.0884 & 2.0226 & & 0.0226 & 1.130 \\
\hline 2.5 & 2.6862 & 2.609 & 2.4745 & & -0.0255 & -1.020 \\
\hline 3.0 & 3.2162 & 3.1316 & 3.0384 & 3.0156 & 0.0156 & 0.520 \\
\hline 3.5 & 3.772 & 3.6737 & 3.5642 & 3.5244 & 0.0244 & 0.697 \\
\hline 4.0 & 4.3352 & 4.2216 & 4.0935 & 4.0352 & 0.0352 & 0.880 \\
\hline 4.5 & 4.9199 & 4.7859 & 4.6325 & 4.5421 & 0.0421 & 0.935 \\
\hline
\end{tabular}

When the branch lines occurs two-phase metal short circuit, the results of distance measurement is shown in Table 2. In the case that the branch line only installed the fault indicators, fault distance can also be relatively accurate measurement.

Tab. 2 Fault location results of branch lines with metal short circuit

\begin{tabular}{|l|l|l|l|l|l|l|}
\hline $\begin{array}{l}\text { fault } \\
\text { distance(km) }\end{array}$ & FTU1 & FTU2 & FTU3 & FTU4 & $\begin{array}{l}\text { absolute } \\
\text { error(km) }\end{array}$ & $\begin{array}{l}\text { relative } \\
\text { error (\%) }\end{array}$ \\
\hline 0.1 & 0.3012 & 0.236 & 0.1647 & 0.1228 & 0.0228 & 22.8 \\
\hline 0.2 & 0.4065 & 0.3408 & 0.2689 & 0.216 & 0.0160 & 8 \\
\hline 0.3 & 0.5138 & 0.4469 & 0.3735 & 0.3393 & 0.0393 & 13.1 \\
\hline 0.4 & 0.6225 & 0.554 & 0.4784 & 0.4438 & 0.0438 & 10.95 \\
\hline $\begin{array}{l}\text { fault } \\
\text { distance }(\mathrm{km})\end{array}$ & FTU1 & FTU2 & FTU3 & FTU4 & $\begin{array}{l}\text { absolute } \\
\text { error(km) }\end{array}$ & $\begin{array}{l}\text { relative } \\
\text { error (\%) }\end{array}$ \\
\hline
\end{tabular}

When the branch lines occurs no-metal short circuit, the results of distance measurement is shown in Table 3 . The increase of short-circuit resistance causes an increase in the load shunt, which causes an increasing ranging error.

Tab. 3 Fault location results of trunk line with no-metal short circuit

\begin{tabular}{|c|c|c|c|c|c|c|}
\hline \multirow{3}{*}{$\begin{array}{l}\text { fault } \\
\text { distance }(\mathrm{km})\end{array}$} & \multicolumn{3}{|c|}{ location results(km) } & \multicolumn{3}{|c|}{ Absolute error(km) } \\
\hline & \multicolumn{3}{|c|}{ Ground Resistance } & \multicolumn{3}{|c|}{ Ground Resistance } \\
\hline & $1 \mathrm{ohm}$ & 3ohm & 5ohm & $1 \mathrm{ohm}$ & 3ohm & 5ohm \\
\hline 0.5 & 0.5239 & 0.4383 & 0.2334 & 0.0239 & -0.0617 & -0.2666 \\
\hline 1.0 & 1.0234 & 0.9685 & 0.8095 & 0.0234 & -0.0315 & -0.1905 \\
\hline 1.5 & 1.5486 & 1.4972 & 1.3405 & 0.0486 & -0.0028 & -0.1595 \\
\hline 2.0 & 2.0282 & 2.0083 & 1.9126 & 0.0282 & 0.0083 & -0.0874 \\
\hline 2.5 & 2.5374 & 2.5172 & 2.4222 & 0.0374 & 0.0172 & -0.0778 \\
\hline 3.0 & 3.0206 & 3.5125 & 2.9371 & 0.0206 & 0.5125 & -0.0629 \\
\hline 3.5 & 3.5322 & 3.5125 & 3.4454 & 0.0322 & 0.0125 & -0.0546 \\
\hline 4.0 & 4.0424 & 4.0117 & 3.9382 & 0.0424 & 0.0117 & -0.0618 \\
\hline 4.5 & 4.5483 & 4.4915 & 4.3987 & 0.0483 & -0.0085 & -0.1013 \\
\hline
\end{tabular}




\section{Conclusions}

Combined with the fault indicators used in the distribution network having a communication function achieve a precise determination of phase fault distance by impedance method, which solves the ranging challenges posed by uneven impedance in hybrid line. The algorithm is validated by ATP simulation. Simulation shows that this method is suitable for complex and diverse distribution network phase short circuit fault location. Its positioning errors are within a reasonable range.

\section{References}

[1] Gonzalez P, Romero E. Experimental tests of High Impedance Faults in MV rural distribution network. Optimization of Electrical and Electronic Equipment, 2014 International Conference on.

[2] Lopes F V, Santos W C. A transient based approach to diagnose high impedance faults on smart distribution networks. Innovative Smart Grid Technologies Latin America (ISGT LA), 2013 IEEE PES Conference On.

[3] Silveira E G, Pereira C. Transmission line fault location using two-terminal data without time synchronization. IEEE Trans. on Power Delivery, 2007.

[4] H. Hizam, P.A.Crossley, etc. Fault section identification and location on a distribution feeder using traveling waves. Power Engineering Society Summer Meeting, pp.1107-1111, 2002.

[5] Jian Tan, Study of transmission line traveling wave fault location, Power System Technology, 29(15), pp.65-70, 2009.

[6] Tao Ji, Tongjing Sun, Bingyin Xu. Pulse Signal Injection Based Faulty Line Detection for Small Current Neutral Grounding System. Proceedings of the CSEE, 26(12), pp.89-93, 2006.

[7] Yueyun Chen, Jian Tan: submitted to Power System Technology, Power System Technology, 30(18), pp.89-92, 2006.

[8] Fen Wang, Yi Kang: submitted to Power System Technology, Power System Technology, 32(15), pp.38-41,2008.

[9] Wang shun Liu, Shaofeng Huang, Power system fault analysis, Beijing: China Electric Power Press, 2010. 